\title{
A Concept of Process Validation in Pharmaceutical Industries
}

\author{
Devendra Kumar Mishra \\ Amity Institute of Pharmacy \\ Amity University Uttar Pradesh Lucknow Campus \\ Lucknow, India
}

\begin{abstract}
The aim of the article is to provide a brief presentation and general review on the criticalness of technical support of the pharmaceutical collecting strategy to ensure the security and minimization of the microbial turn of events. The process validation in the manufacturing unit is highly important to verify the surety at each and every stage of production. The microbial danger to aseptically created things in pharmaceutical clean rooms can be reviewed by the use of significant conditions that model the dispersing, move and explanation of microbial contamination, and the usage of numerical characteristics or risk descriptors. This should be conceivable in two-stages, with the primary stage used to overview the trading of contamination from the whole of the sources inside the clean room suite and the resulting stage used to assess both air and surface contact pollution inside fundamental creation locales. These two systems can be used to assess and diminish microbial danger at the basic structure period of the clean room and related collecting processor, brilliantly, for a developed gathering movement. Along these lines, underwriting is a basic piece of value confirmation. This separates the need for pharmaceutical and underwriting, the different ways of thinking, methods, and steps to be checked during the gathering process.
\end{abstract}

Keywords:- Good Manufacturing Practice, cGMP, Process Validation, Agar Medium, Microbial Cleanliness.

\section{INTRODUCTION}

A GMP is called Good Manufacturing Practices. GMP is a framework that guaranteeing that things are constantly passed on and obliged by quality measures and rules, which secure the patient. GMP covers all pieces of creation from the earliest starting point materials, premises, and equipment to the arrangement and individual tidiness of staff [1]. Beyond the initiatives of industry, requirements for licensing and current good manufacturing practice (cGMP) mandate that the systems and procedures which ensure the purity, potency, efficacy, and viral safety of such products are in place, maintained, and rigorously enforced. The U.S. FDA gives managerial oversight of these requirements through the approval of processes and equipment, and thorough inspections of facilities and manufacturing practices. The agency has varied and significant authority to ensure compliance with these regulations [2]. For viable globalization of the normal things, makers need to meet the close by GMP rules and display great pre-and post-promote

\author{
Shubham Shukla \\ School of Biomedical and Pharmaceutical Sciences \\ Babasaheb Bhimrao Ambedkar University \\ Lucknow, India
}

assessment before bargains [3]. The improvement of a prescription thing is a broad technique that incorporates quiet disclosure, research office testing, the animal looks at, clinical primers, and authoritative enlistment. This long strategy is imperative to ensure the sufficiency and security of the prescription thing [4]. In the mid of 1970s, there were two FDA authorities who proposed the possibility of endorsement to improve the idea of pharmaceuticals, and the name of them are Bud Loftus and Ted Byers. The most critical and seen limits of CGMPs are the process validation in pharmaceuticals [5]. The necessities of approach underwriting show up in the quality structure $(\mathrm{QS})$ rule. The objective of a quality structure is to constantly pass on things that are fit for their ordinary use. The endorsement is the place it incorporates people from various controls of the plant [6]. In the various human activities scaled as well as downscale, the animals in the earth address, a concealed anyway dangerous peril factor. Concern has extended with the introduction of bleeding-edge advancements in centers, industry, and cultivating [7]. The microbial danger to aseptically created things in pharmaceutical clean rooms can be reviewed by the use of significant conditions that model the dispersing, move and explanation of microbial contamination, and the usage of numerical characteristics or risk descriptors, it is portrayed in regulatory records conveyed by masters that join the FDA in the US [8] and European Commission [9]. Microorganisms possess the ability to disseminate and adopt various environmental conditions even harsh and extreme conditions. This advantage for microbial life persistence is a serious problem for any organization working in the healthcare industry, notably pharmaceutical firms [10].

Some Definition of Validation:

\section{$>$ As shown by the FDA [11]}

Affirmation of thing quality is gotten from wary and central thought with respect to different hugeness factors, including the decision of significant worth process through in-process and completed outcome testing.

\section{As shown by the US FDA in 1978 [12]}

They had stated that it is conveyance processes that display what to do and also what it reason or is tended to do. The affirmation of it is gotten through the course of action and verification of information, ideally, starting from the process progress stage and proceeding with the creation stage. Also they stated that the validation from a general perspective joins processability (the limit of materials, hardware, framework, building, staff), yet it besides 
remembers the control for the whole fundamental for repeated groups or runs.

\section{$>$ European Commission - 2000}

Validation point by point proof that the system worked inside developed limits, can able to do enough and the same required to make a remedial thing meeting its destined decisions and quality traits [13].

WHO has portrayed some of the guidelines for the validation and it's represented a display of demonstrating that any methodology, process, gear, material, improvement, or framework really prompts the run of the typical results. Validation showing of illustrating, incomprehension of GMPs, which have any process, truly prompts foreseen results. The documented check that the process worked with in setting up cutoff points can perform sensibly reproducibly to make an accommodating thing meeting its destined focal points and quality attributes.

\section{NEED OF VALIDATION}

The pharmaceutical business uses exorbitant material, present-day workplaces, and sorts of apparatus and particularly qualified personals. Point by point study and control of the storing up process bunch validation is essential if dissatisfaction cost is to be decreased and profitability is improved. On the off chance that it would not be achievable to utilize gear not knowing whether it will make the thing we need, not to utilize the individuals with no affirmation that they can do or dismissal to execute process checks or examination to guarantee that thing meet nuances. The reasonable use of these favorable circumstances is noteworthy for the proceeded with the achievement of the business. The expense of thing bafflement rejects, fixes up, overviews, and battles are an adequate piece of full-scale creation cost [14].

\section{$>$ Different stages in Process Validation}

Stage 1: The first stage of this process is known to be Pre-validation Ability Stage which covers all exercises identifying with thing imaginative work, plan pilot bunch reviews, scale-up analyzes, move of headway to business scale packs, setting up unwavering quality conditions and cutoff, and treatment of in-process and completed estimation structures, gear limit, establishment limit master creation record, operational capacity and process limit [15].

Stage 2: The second stage of this process is known to be the process validation stage. It is relied upon to avow that each settled impediment of the crucial process limit is significant and that agreeable, weather the all parameters were followed or not, and if followed then it was it must be in a proper sequence [13].

Stage 3: The third stage of this process is called as the validation upkeep Stage. This stage requires different parameters like the visit investigation of all fundamentals related records, also to ensure the review the data of validation, to guarantee that there have been no mismatch, alteration, deviations, changes in the parameter and acclimations to the creation process and that all SOPs, including methods of change control, which have been followed in the process and all parts or bundles passed on will followed to their typical nuances, and it will ensure the safety and efficacy towards the complete ongoing processes and enhance the quality of the product [6].

\section{TYPES OF VALIDATION}

\section{A. Analytical Validation}

It is defined as the assessment of thing quality traits through investigation, to show reliability is being kept up all through the thing life cycle and that the accuracy, precision, unequivocally, selectivity, LOD, flawlessness, quality, linearity, and detail has not been sabotaged.

\section{B. Equipment Validation}

It is defined as the confined into insight level, Operational Capacity (OQ), and Execution Capacity (PQ). A degree of knowledge reports decides the static qualities of an office or thing to exhibit that the establishment of the unit has been sufficiently performed and the establishment focal points of the producer have been met.

\section{Process Validation}

It is defined as the documented methodology which gives a veritable level of confirmation that a particular process will reliably make a thing meeting its ordained detail and quality properties.

It has been divided into various sorts as follows:-

Prospective validation: It has been depicted as the foundation of archived affirmation that a structure does what its inspiration to do expose to preplan appear. Along these lines to manage validation is customarily endeavored at whatever points another condition, process, or office must be insisted before business routine pharmaceutical specifying begins.

Retrospective validation: It has been depicted as the foundation of uncovered check that a framework does what its inspiration to do dependent on audit and assessment of recorded information. This is developed by the outline of the chronicled conveying testing information to show that the process has dependably stayed in charge.

Concurrent validation: It takes after unavoidable, next to the working firm will sell the thing during the limit runs, to people all around at its market cost. This validation recalls for process seeing of major processing steps and thing evaluated. It is the accentuation of a process for validation or a particular piece of it.

Revalidation: It is routinely performed to the accreditation of an ideal time validation for an uncommon survey. It provides a brief idea for the check of those changes in a process and/the process conditions that are introduced don't forebodingly influence process properties and thing quality. 


\section{Process/Product Validation}

It is intended to reveal a check which gives genuine information of affirmation that a particular structure which dependably settle on a thing interacting its destined choices and characteristics of the quality [16].

\section{MATERIAL AND METHODS}

For any bacterium to be incited under any circumstances it is essential to give the appropriate biochemical and biophysical condition. The biochemical (dietary) condition is made available as a culture medium. The fixings in culture media run from unadulterated manufactured blends to complex materials, for instance, think or procedures of plant and animal tissues [17]. If all the components of a culture medium are known, both abstractly and quantitatively, the medium is known as a falsely portrayed medium. These media are of uncommon impetus in inspecting the fortifying necessities of microorganisms or in thinking about an amazing collection of their metabolic activities. In a bewildering medium, that cautious mixture creation isn't known, and such a medium is consistently masterminded from complex materials, e.g., body fluids, tissue concentrates and implantations, and peptone [18]. Then for the assessment of the experiment requires some of the equipment like weighing balance for the proper scaling of weight for the preparation of media, autoclave for proper sterilization of equipment, microbial colony counter to detect and count the colony formation, hot air oven for sterilization of glassware and other, laminar airflow for the transplantation of sample in media to minimize another bacterial attack, an incubator for the development of microbial growth under its favorable condition. [19].

\section{A. Method for collection of samples}

Taken three pieces of cotton in a small portion and it was wet with purified water, and then it was swipe on three different surfaces, i.e.; (1) from the normal floor surface, (2) from the nearby surface of hot air oven, and (3) from Laminar Air Flow bench.

\section{B. Nutrient Agar Media}

Nutrient Agar Media is a liquid medium commonly used for the cultivation of microorganisms that are nondemanding in their nutritional requirements e.g. water-borne organisms, air, soil, and dust flora. It was used for the cultivation and determination of bacteria [20].

\begin{tabular}{|c|c|c|}
\hline S.No & Ingredients & Grams/Liter \\
\hline 1. & Peptone & 5.0 \\
\hline 2. & Sodium Chloride & 5.0 \\
\hline 3. & Beef extract & 1.5 \\
\hline 4. & Yeast extract & 1.5 \\
\hline 5. & Agar & 15 \\
\hline 6. & Distilled water & $1000 \mathrm{ml}$ \\
\hline
\end{tabular}

Table 1:- Components of medium
The ingredients were weighed and dissolved in $800 \mathrm{ml}$ of water one by one.

$>$ The mixture was boiled and the medium was dissolved completely.

$>$ Then it was cooled to $50^{\circ} \mathrm{C}$ and adjusted the $\mathrm{pH}$ with the help of $\mathrm{pH}$ meter, and then made up the volume to $1000 \mathrm{ml}$.

$>$ Then the solution was dispensed into flasks and the mouth of it was covered with the help of cotton and aluminum foil.

$>$ It was sterilized by autoclaving at $15 \mathrm{lbs}$ pressure $\left(121^{\circ} \mathrm{C}\right)$ for 15 minutes.

C. Procedure for determination of microbial growth

$>$ All the needed glassware was washed and dried.

$>$ Taken samples, sterilized glassware, and prepared sterilized nutrition agar media in the aseptic area.

$>$ Firstly the working area of the laminar airflow bench was cleaned with the help of $70 \%$ isopropyl alcohol.

$>$ Then the media flasks were unwrapped and its mouth was sterilized with the help of flame (It was done to reduce the chance of impurity).

$>$ Then differentially collected samples water was dropped in respective petridish and poured the media on it, and then it was kept on the side to solidify the agar.

$>$ Then petridish was covered and inverted to reduce the excess of media.

$>$ Petridish was then kept for incubation in the incubator for $24 \mathrm{hrs}$.

\section{EVALUATION OF MICROBIAL GROWTH}

After 24hrs when petridish was removed from incubator then it was observed under "Microbial Colony Counter" to find the microbial growth in different petrdishes and marked the different colonies formed by the microbial growth.

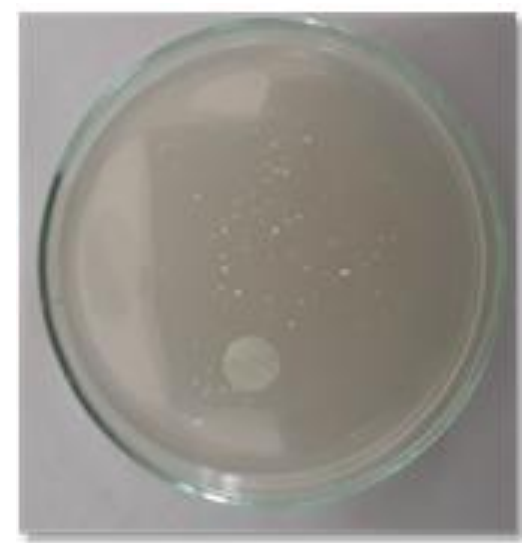

Fig 1:- Petridish 1

(Sample was taken from floor) 


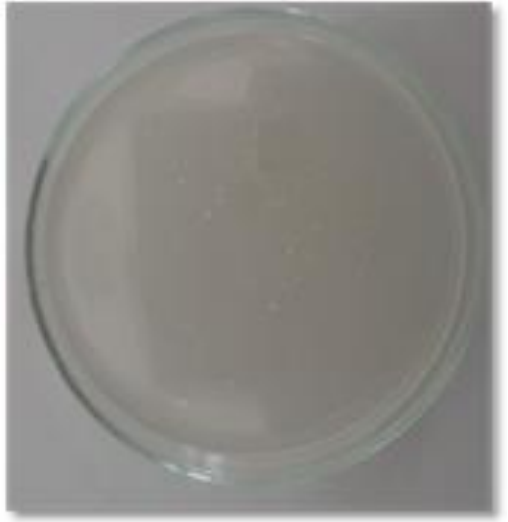

Fig 2:- Petridish 2

(Sample was taken from near hot air oven)

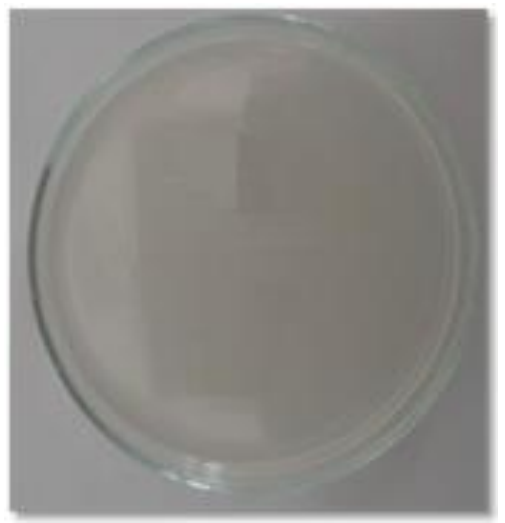

Fig 3:- Petridish 3

(Sample was taken from Laminar air flow bench)

\section{RESULT AND DISCUSSION}

On observing all three different petridishes, the results are as follows:

$>$ The sample collected from the floor (here referred to as Sample 1) has the highest number of microbial growth (microbial colonies).

$>$ The sample collected from near hot air oven (here referred to as Sample 2) has the lowest number of microbial growth (microbial colonies).

$>$ The sample collected from the laminar airflow bench (here referred to as Sample 3) does not form any microbial growth (microbial colonies).

$>$ The growth of microbes or formations of bacteria's colony is not similar in each petridishes, because:

$>$ The sample collected from the floor contains a high amount of microbial exposure because of high humidity, favorable temperature, and high contamination due to different sources like soil which came from footwear or by another mean, and the soil is highly responsible or favorable for the bacterial growth. Thus, when the cotton swaps from the floor it includes the maximum number of the microbes.
It can be controlled with the help of proper cleaning with disinfectant and precautions should be taken for other contamination.

$>$ The sample collected from near hot air oven contains a low amount of microbial exposure compared to the floor because there is no chance of contamination by footwear, and due to the release of heat by hot air oven. But there is microbial growth due to sudden Accidently falls of the product during loading and unloading in a hot air oven.

$>$ It can be controlled by proper handling of products during loading and unloading in a hot air oven and clean the area with disinfectant.

The sample collected from the laminar airflow bench does not possess microbial growth because laminar flow contains UV light, HEPA filter which is highly effective against bacterial growth and cleaning off the bench with alcohol results to no bacterial growth.

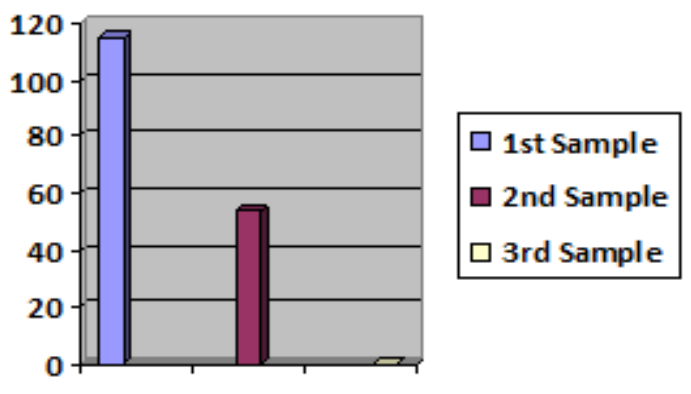

Chart 1:- Microbial Growth in Media

\section{CONCLUSION}

This provides a comprehensive knowledge about the Good Manufacturing Practices, Validation, and cleaning of various instruments followed in different industries or departments, and as well as it provides vast knowledge for cleaning in microbial section to prevent any chance of microbial growth or contamination due to any mean. The microbial section provides an environment for the hygiene or sterilized function in any department which helps to authenticate the reliability and quality of any particular products, thus microbial section should be properly cleaned and the equipment should be functions at its best because the microbial section is very susceptible or prone to be contaminated with the external environment and get affected with a microbial attack if the cleaning of it doesn't do properly. In this project work, the quality of cleaning in the microbial section was tested with taking reference to other places and found that the quality of the microbial section was much better or said to be best from others due to proper cleaning, arrangement, and equipment which provide a sterilized environment. So the cleaning is a prior fundamental property of any product sterility and to minimize or prevent any chance to the growth of microbes or contaminations which can affect the products. 


\section{REFERENCES}

[1]. T.P. Dongare, GMP in Pharmaceutical Industry Global cGMP and regulatory expectations, First Edition, PharmaMed Press: New Delhi, 2018.

[2]. JP. Ways, MS. Preston, D. Baker, J. Huxsoll, J. Bablak, "Good manufacturing practice (GMP) compliance in the biologics sector: plasma fractionation," Biotechnol Appl Biochem, vol. 30, pp. 257-265, 1990.

[3]. DR. Briggs, "The regulation of herbal medicines in Australia," Toxicology, vol. 181, pp. 565-570, 2002.

[4]. USPNF. The United States Pharmacopeia XXIII and the National Formulary XVIIl. Rockville, MD: The United States Pharmacopoeia1Convention, Inc.; 1995.

[5]. ME. Aulton, Pharmaceutics-The Science of Dosage Form Design. 2nd ed, Elsevier: Churchill Livingstone, 2006.

[6]. H. Kaur, G. Singh, N. Seth. "Pharmaceutical Process Validation: A Review," Journal of Drug Delivery \& Therapeutics, vol. 3(4), pp. 189-194, 2013.

[7]. C. Pasquarella, O. Pitzurra, A. Savino. "The index of microbial air contamination," J Hosp Infect, vol. 46(4), pp. 241-256, 2000.

[8]. Food and Drug Administration. Guidance for Industry: Sterile Drug Products Produced by Aseptic Processing - Current Good Manufacturing Practice. Silver Spring, MD, USA: FDA; 2004.

[9]. European Commission. EudraLex. The Rules Governing Medicinal Products in the European Union. Volume 4: EU Guidelines to Good Manufacturing Practice - Medicinal Products for Human and Veterinary Use. Annex 1 - Manufacture of Sterile Medicinal Products. Brussels, Belgium: European Commission; 2008.

[10]. L. Clontz, Microbial limit and bioburden tests: validation approaches and global requirements, 2nd ed., CRC Press; Boca Raton, 2009.

[11]. RA. Nash, AH. Wachter, Pharmaceutical Process Validation an International 3rd ed. Revised and Expended, Marcel Dekkar, Inc: New York, 2003.

[12]. RC. Patel, CK. Bhuva, RP. Singh, A. Dadhich, A. Sharma, Pharmaceutical Process Validation, Pharmatutor -ART- 1053.

[13]. E. Jatto, OA. Okhamafe, "An overview of pharmaceutical validation and process controls in drug development," Tropical J pharm research, vol. 2, pp.115-122, 2004.

[14]. Guidance for Industry: Process Validation:General Principles and Practices. U.S. Department of Health and Human Services, Food and Drug Administration, Centre for Drug Evaluation and Research (CDER), Centre for Biologics Evaluation and Research (CBER), Centre for Veterinary Medicine (CVM), January 2011.

[15]. PR. Ram, S. Saroj, L. Shreekrishna, P. Priyanka, “A Review On Pharmaceutical Process Validation Of Solid Dosage Form [Tablets]," Journal of Drug Delivery \& Therapeutics, vol. 5(6), pp. 1-7, 2015.

[16]. USA-FDA, Guidelines on General Principles of Process Validation, CEDR, FDA-USA, 1987.
[17]. KE. Clark, EL. Boston, R. Brent, "Media Preparation and Bacteriological Tools," Current Protocols in Molecular Biology, pp.1.1.1-1.1.7, 2002.

[18]. FC. Neidhardt, PL. Bloch, DF. Smith. "Culture Medium for Enterobacteria," Joural of Bacteriology, vol. 119(3), pp. 736-747, 1974.

[19]. MA. Durivage, The certified Pharmaceutical GMP Professional Handbook, 2nd ed, American Society for Quality, Quality Press: Milwaukee, 2016.

[20]. IO. Ogbonna, "Nutrient Compositions of Different Grains for Use in the Formulation of Bacteriological Media," The International Journal of Biotechnology, vol. 6(1), pp. 1-10, 2017. 